Vernakuläre Wissenschaftskommunikation 


\section{Lingua Academica}

Beiträge zur Erforschung historischer Gelehrtenund Wissenschaftssprachen

Herausgegeben von

Wolf Peter Klein, Michael Prinz und Jürgen Schiewe

Wissenschaftlicher Beirat

Ulrich Ammon (Duisburg-Essen), Marian Füssel (Göttingen), Daniel Fulda (Halle), Michael D. Gordin (Princeton), Mechthild Habermann (Erlangen),

Marion Gindhart (Mainz), Thomas Gloning (Gießen), Angelika Linke (Zürich/

Linköping), Leo Kretzenbacher (Melbourne), Uwe Pörksen (Freiburg),

Ulrich Johannes Schneider (Leipzig), Dirk Werle (Heidelberg)

\section{Band 1}




\section{Vernakuläre \\ Wissenschaftskommunikation}

Beiträge zur Entstehung und Frühgeschichte der modernen deutschen Wissenschaftssprachen

Herausgegeben von

Michael Prinz und Jürgen Schiewe 
Die Druckvorstufe dieser Publikation wurde vom Schweizerischen Nationalfonds zur Förderung der wissenschaftlichen Forschung unterstützt.

ISBN 978-3-11-047498-5

e-ISBN (PDF) 978-3-11-047695-8

e-ISBN (EPUB) 978-3-11-047503-6

\section{(cc) BY-NC-ND}

Dieses Werk ist lizenziert unter der Creative Commons

Attribution NonCommercial-NoDerivatives 4.0 Lizenz. Weitere Informationen finden Sie unter http://creativecommons.org/licenses/by-nc-nd/4.0/.

\section{Library of Congress Publication Number: 2018956850}

\section{Bibliografische Information der Deutschen Nationalbibliothek}

Die Deutsche Nationalbibliothek verzeichnet diese Publikation in der Deutschen Nationalbibliografie; detaillierte bibliografische Daten sind im Internet über http://dnb.dnb.de abrufbar.

(C) 2018 Michael Prinz und Jürgen Schiewe, publiziert von Walter de Gruyter GmbH, Berlin/Boston

Dieses Buch ist als Open-Access-Publikation verfügbar über www.degruyter.com, https://www.doabooks.org und https://www.oapen.org

Satz: Meta Systems Publishing \& Printservices GmbH, Wustermark Druck und Bindung: CPI books GmbH, Leck

www.degruyter.com 